\title{
Optical measurement of the thickness of an individual layer in a plastic multilayer compound
}

\author{
Huber Michael", Weber Wolfgang* (w.weber@sensorik-bayern.de), Plohmann Daniel*, Steigerwald \\ Hubert ${ }^{\# *}$ \\ \#) Strategische Partnerschaft Sensorik e.V., Regensburg; *) Sensorik-Bayern GmbH, Regensburg
}

Appropriate topic number: G.4: Optical inspection, in-line quality assurance

In plastic multilayer compounds the functionality of the system is often provided by a single functional layer. This is the case for food packaging materials, floor heating or gas pipes for example. The functional layer alone is responsible for the functional features of the system, e.g. diffusion impermeability, and is deposited on a cheap plastic substrate like PP, PVC or PE using an adhesive. In such systems, the thickness of the substrate typically exceeds the thickness of the other components considerably. The materials used for these functional layers often are comparatively expensive, which makes it desirable to have these layers as thin as possible.

The pipe extrusion method is the standard manufacturing technique for the production of multilayer plastic pipes. Within this process variations of the thickness of the various layers can occur. Therefore, to be on the safe side, the functional layer is deposited with a significantly higher thickness than actually required; otherwise the thickness variations could result in the loss of the functionality. This procedure causes a significantly higher material consumption and financial effort. Currently an online regulation of the thickness of the functional layer is not possible, as no available sensor can selectively measure the thickness of the single layers.

Here, we present an optical solution to this problem. The solution is based on the absorption principle: Every material has a specific absorption spectrum for electromagnetic radiation. This absorption spectrum is comparable to a finger print, which can be used to identify materials. While plastics cannot be distinguished using standard methods that are based on e.g. dielectric properties, their absorption spectra often are significantly different, particularly in the NIR up to THz regime, where various rotational or vibrational modes are excited. The corresponding absorption spectra mainly depend on the molecular consistency of the substance. As a first step, one needs to obtain the individual absorption spectra of the materials involved. The operating frequency is chosen in a way that the absorption of the functional layer is significantly higher than the absorption of the other layers at that frequency. If a spectral analysis is performed on a sufficiently large frequency range, there is a high probability of identifying a suitable operating frequency. The case presented here deals with floor heating pipes for which we have found several possible operating frequencies.

Thus an electromagnetic wave at the operating frequency is absorbed mainly by the functional layer of the pipe and the signal that can be detected depends almost exclusively on the thickness of this layer. As the absorption coefficient depends exponentially on the thickness of the layer, the resolution of this type of measurement is very high. Depending on the boundary conditions, a resolution in the lower micrometer regime can be realized. Certain operating frequencies may require the use of extraordinary optical sources like quantum cascade or distributed feedback laser sources for this technique to work.

The main focus of the talk will be set on floor heating pipes. We present the realization of the sensor system including a mechanical device that allows moving the sensor system around the pipes with a circular cross section and includes components like lenses to give the beam a defined profile.

Our solution is designed for the quality management process of conventional multilayer compounds like floor heating pipes. Thickness deviations of the monitored layer can be detected during the pipe extrusion process, therefore making it no longer necessary to apply excessively thick layers of the expensive functional material without compromising the quality of the product.

Acknowledgement: This project is supported by the "Zentrales Innovationsprogramm Mittelstand (ZIM), Förderkennzeichen DLEP10040 
Figures:

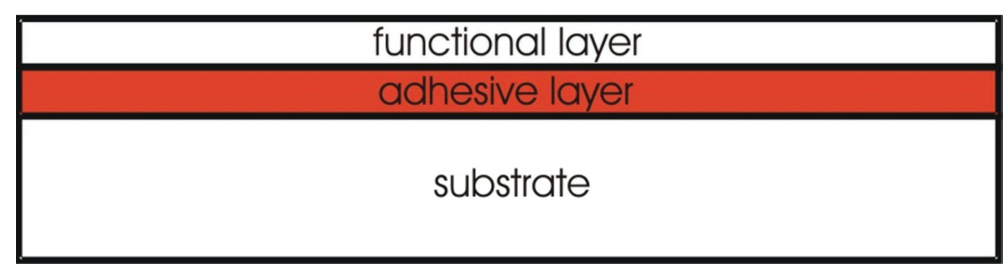

Figure1: Schematic illustration of a typical structure of multilayer plastics. The functional layer is deposited on a substrate using an adhesive and provides the required properties of the system. Here, we want to present a method to selectively measure the thickness of this layer and relate to the specific case of floor heating pipes during the extrusion process. 